\title{
Impact of initial pulse characteristics on the mitigation of self-phase modulation by sinusoidally time varying phase
}

\author{
Sonia Boscolo ${ }^{1}$, Frédéric Audo ${ }^{2}$ and Christophe Finot ${ }^{2, *}$ \\ ${ }^{1}$ Aston Institute of Photonic Technologies, School of Engineering and Applied Science, Aston \\ University, Birmingham B4 7ET, United Kingdom \\ ${ }^{2}$ Laboratoire Interdisciplinaire Carnot de Bourgogne, UMR 6303 CNRS-Université de \\ Bourgogne-Franche-Comté, 9 avenue Alain Savary, BP 47870, 21078 Dijon Cedex, France \\ * Corresponding author: \\ E-mail address: christophe.finot@u-bourgogne.fr \\ Tel.: +33 380395926
}

\begin{abstract}
A simple and efficient approach to suppress undesirable self-phase modulation (SPM) of optical pulses propagating in fiber-optic systems is based on imposing a sinusoidal temporal phase modulation on the pulses to offset the chirp generated by SPM (Audo et al. 2017). Here, we present a detailed analysis of this method. We derive an exact formula for the reduction of the SPM-induced rms spectrum broadening of an initially Gaussian pulse enabled by the sinusoidal compensation, and we assess the effects of the initial pulse shape and duration on the effectiveness of the technique by means of numerical simulation. The differences between pre- and postpropagation compensation schemes are also discussed.
\end{abstract}

Keywords: Self phase modulation; nonlinear fiber optics; pulse shaping 


\section{Introduction}

The propagation of intense ultra-short optical pulses in a Kerr medium such as an optical fiber still remains a critical issue for the performance of many optical systems such as beam delivery, optical communication or pulse amplification systems. This is because the self-phase modulation (SPM) of the propagating pulse usually causes a broadening of the pulse spectrum that is typically accompanied by an oscillatory structure covering the entire frequency range (Stolen,Lin 1978; Agrawal 2006; Wang et al. 1994). Several strategies have been proposed and successfully deployed to counteract the deleterious effects of SPM in fiber-optic systems (Richardson et al. 2010). The most basic one consists in spectrally filtering the broadened spectrum after nonlinear propagation. However, this approach is power inefficient and may induce large distortions of the pulse temporal profile. More relevant approaches rely on spatial or temporal scaling to reduce the impact of nonlinearity. These include the use of very large mode area fibers or hollow core fibers (Ma et al. 2014; Michieletto et al. 2016), the well-known technique of chirped pulse amplification (CPA), in which ultra-short laser pulses are stretched in time prior to amplification and recompressed back to ultra-short pulse duration after amplification through the reversal process of stretching (Strickland,Mourou 1985), and new schemes for pulse division amplification (Seghilani et al. 2017). A different class of approaches tends to exploit the nonlinear properties of the propagation. For example, when the pulse launched in the fiber has a hyperbolic secant shape and its power and duration are correlated, the effects of SPM and anomalous second-order groupvelocity dispersion (GVD) can cooperate in such a way that the pulse either does not change along the fiber length or follows a periodic evolution pattern (Hasegawa,Tappert 1973; Agrawal 2006; Ouzounov et al. 2003). A class of optical solitons arising from the interaction of negative fourthorder dispersion and SPM in photonic crystal waveguides has also been recently demonstrated (Blanco-Redondo et al. 2016). However, the generation of solitons imposes very stringent conditions on the pulse and fiber/waveguide properties. Other approaches making the most of the Kerr nonlinearity include the exploitation of the peculiar properties of parabolic shaped pulses and self-similar evolution (Finot et al. 2009; Pierrot,Salin 2013), the use of other types of pre-shaped input pulses, and the compensation of nonlinear phase shifts with third-order dispersion (Shah et al. 2005; Zhou et al. 2005). However, none of these last techniques preserves the pulse temporal duration.

A simple technique to compensate the nonlinear phase due to SPM and related spectrum 
broadening of nanosecond or picosecond optical pulses consists in using an electro-optic phase modulator to impart the opposite phase to the pulses. This method, which emulates the use of a material with a negative nonlinear index of refraction, has proved to be successful in fiber-optic and free-space optical telecommunication applications using phase-shift keying systems (Xu et al. 2002; Ulmer et al. 2016), and in the generation of high-peak-power nanosecond pulses (Su et al. 2013; Munroe et al. 2009). Moreover, the technique can be integrated into existing CPA systems to deal with femtosecond pulses (van Howe et al. 2006; Zhu et al. 2007). We have recently demonstrated that for Gaussian shaped input pulses, the use of a simple sinusoidal drive signal for the phase modulator with appropriate amplitude and frequency is sufficient to reduce the nonlinear spectrum broadening to a large degree, and to significantly enhance the spectral quality of the pulses while their temporal duration remains unaffected (Audo et al. 2017). We have also studied the use of a sinusoidal phase compensation for improving the extinction ratio of signals impaired by a coherent background (Audo,Finot 2017).

In this paper, we present a comprehensive analysis of the SPM-mitigation method involving the use of a sinusoidal phase modulation. Most of the previous works are primarily experimental in nature (van Howe et al. 2006; Zhu et al. 2007; Xu et al. 2002; Ulmer et al. 2016; Su et al. 2013; Munroe et al. 2009) and, for instance, have not discussed the sensitivity of the technique to the initial pulse characteristics in detail. First, we recall the concept of our method and the situation being studied. Next, we present an exact closed formula for the rms spectral width of an initially Gaussian pulse after undergoing SPM and with the corrective phase applied, which confirms the substantial reduction of the SPM-induced spectrum broadening attainable with the phase compensation. Then, we describe the impact of the initial pulse shape and duration on the effectiveness of the technique through numerical simulation of the governing equation. A comparison of pre- and post-propagation compensation schemes is also provided. 


\section{Principle of operation and situation under investigation}

Pulse propagation in an optical fiber is modeled by the standard nonlinear Schrödinger equation (NLSE) (Agrawal 2006):

$$
i \frac{\partial \psi}{\partial z}=\frac{1}{2} \beta_{2} \frac{\partial^{2} \psi}{\partial t^{2}}+\gamma|\psi|^{2} \psi
$$

where $\psi(z, t)$ is the complex envelope of the pulse, $z$ is the propagation coordinate, $t$ is the retarded time, $\beta_{2}$ is the GVD parameter, and $\gamma$ is the coefficient of Kerr nonlinearity of the fiber. When the effects of the Kerr nonlinearity are considered over those distances and power levels such that we can neglect dispersion, the solution to Eq. (1) is simply $\psi(z, t)=\psi(0, t) \exp i\left[\varphi_{N L}(z, t)\right]$, where $\psi(0, t)$ is the field amplitude at $z=0$, and $\varphi_{N L}(z, t)=B I(t)$ is the SPM-induced nonlinear phase shift. Here, $I(t)=|\psi(0, t)|^{2} / P_{0}$ is the (longitudinally unchanging) intensity profile of the pulse, $P_{0}$ is the peak pulse power, and $B=\gamma P_{0} z$ is the maximum phase shift that occurs at the pulse center located at $t=0$ and is widely known as the $B$-integral. In the presence of loss or gain, the physical propagated length $z$ is replaced with an effective length defined as $L_{\text {eff }}=[1-\exp (-\alpha z)] / \alpha$, where $\alpha>0(<0)$ accounts for loss (gain) (Agrawal 2006). For an initially transform-limited pulse, the presence of a temporal variation of the instantaneous frequency (chirp) $\delta \omega_{N L}(t)=-\mathrm{d} \varphi_{N L} / \mathrm{d} t$ causes broadening of the pulse spectrum over its initial width at $z=0$, while the nonlinear nature of the chirp leads to spectral oscillations.

In order to illustrate our idea and provide some simple analytic design rules, we consider an initial chirp-free pulse with a Gaussian temporal intensity profile: $I_{G}(t)=\exp \left(\neg t^{2} / T_{0}{ }^{2}\right)$, where $T_{0}$ is the $1 / e$ intensity half-duration, propagating in a highly nonlinear fiber. The initial pulse and fiber parameters used in the numerical simulation of Eq. (1) are: full-width at half-maximum (fwhm) pulse duration $=100 \mathrm{ps}, P_{0}$ in the Watt range, fiber length $=500 \mathrm{~m}, \gamma=10 / \mathrm{W} / \mathrm{km}$ and $\beta_{2}=$ $3 \cdot 10^{-3} \mathrm{ps}^{2} / \mathrm{m}$ (Audo et al. 2017). With such parameters, the nonlinearity-dominant regime of propagation is applicable. Figure 1 shows the pulse characterizations at the fiber output for a $B$ integral accumulated over the fiber length of $B=2 \pi$ rad. We can see that the SPM-induced frequency chirp $\delta \omega_{N L}$ has a non-monotonic temporal variation across the pulse with two extrema around the pulse center (Fig. 1(a), red curve). The time separation between these extrema and the 
corresponding frequency excursion can be computed by $\Delta \tau=\sqrt{2} \cdot T_{0}$ and $\Delta \omega=B \sqrt{2} \exp (\neg 1 / 2) / T_{0}$, respectively (Agrawal 2006). The pulse spectrum has been significantly broadened over its initial width by the SPM-generated frequency components and has developed a multi-peak structure as a result of interference between distinct points in the pulse having the same instantaneous frequency (Fig. 1(b)).

A common approach to transform a chirped pulse into a close-to-Fourier-transform-limited pulse consists in customizing the pulse spectral phase by applying a parabolic phase profile or more complex profiles in the frequency domain. The temporal intensity profile of the pulse is significantly modified with this approach and features a much shorter duration whereas the pulse spectrum remains unaffected. The SPM-compensation method that we study here is radically different as the phase of the pulse is modified directly in the time domain. By imparting to the pulse a phase shift that is opposite to the nonlinear phase shift, the spectrum broadening caused by SPM can be completely reversed (Xu et al. 2002; Munroe et al. 2009; Su et al. 2013). In principle, if the pulse shape is known, the appropriate drive signal for the phase modulator can be synthesized. However, because this approach entails high optoelectronics bandwidth requirements it is of significant practical importance to minimize the optoelectronics bandwidth that is involved. In the context of the transmission of telecommunication signals, it has been shown that approximating the signal waveform with just one or two harmonics suffices to achieve a significant amount of SPM compensation (Xu et al. 2002; Ulmer et al. 2016; Zhu et al. 2007). This can also be expected from our results in Fig. 1(a): the observed time dependence of the SPM-induced frequency chirp of the Gaussian pulse indeed suggests that this chirp can be offset by driving the phase modulator with the simplest waveform that can be synthetized electronically, i.e. a sinusoid: $\varphi_{C}(t)=A_{C} \cos \left(\omega_{C} t+\phi_{0}\right)$, producing the chirp $\delta \omega_{c}=A_{C} \omega_{C} \sin \left(\omega_{C} t+\phi_{0}\right)$. An effective cancellation of the nonlinear phase imposes the phase shift $\phi_{0}=\pi$. Note that inappropriate values of $\phi_{0}$ such as in-phase modulation $\left(\phi_{0}=0\right)$ may lead to enhanced spectral broadening instead of the targeted SPM-cancelation. Therefore, the temporal delay between the electrical modulation driving the phase modulator and the train has to be controlled. This also adds some constraints on the choice of $\omega_{c}$ with respect to the repetition rate $\mathrm{R}$ of a pulse source so that $\omega_{c} /(2 \pi R)$ should be an integer. Inspired by a previous numerical work relating to the optimization of the SPM-driven spectral compression process (Boscolo et al. 2016), we choose the angular frequency and amplitude of the modulation for the Gaussian pulse as (Audo et al. 2017): 


$$
\left\{\begin{array}{l}
\omega_{C}=\omega_{C T G}=\pi / \Delta \tau=\pi /\left(\sqrt{2} \cdot T_{0}\right) \approx 2.221 / T_{0} \\
A_{C}=A_{C T G}=\Delta \omega / \omega_{C T G}=2 \cdot B \cdot \exp (-1 / 2) / \pi \approx 0.386 \cdot B
\end{array}\right.
$$

With this parameter choice, the external phase modulation indeed compensates the nonlinear phase accumulated by the pulse to a large degree: the total chirp, $\delta \omega_{c}+\delta \omega_{N L}$, is now canceled almost completely over the central region of the pulse (Fig. 1(a), blue curve). This entails a significantly narrower spectrum that is now close to its original width (Fig. 1(b1)). Small pedestals are still present in the spectrum, but their intensity level is below $20 \mathrm{~dB}$. A measure of how close a pulse is to its Fourier transform limit is provided by the spectral Strehl ratio, defined as the ratio of the power spectral density (PSD) of the actual pulse to the PSD obtained assuming a transform-limited pulse. While after nonlinear propagation in the fiber, the maximum relative PSD of the pulse spectrum has dropped to 0.22 the application of the external phase modulation re-establishes a Strehl ratio of 0.73 .
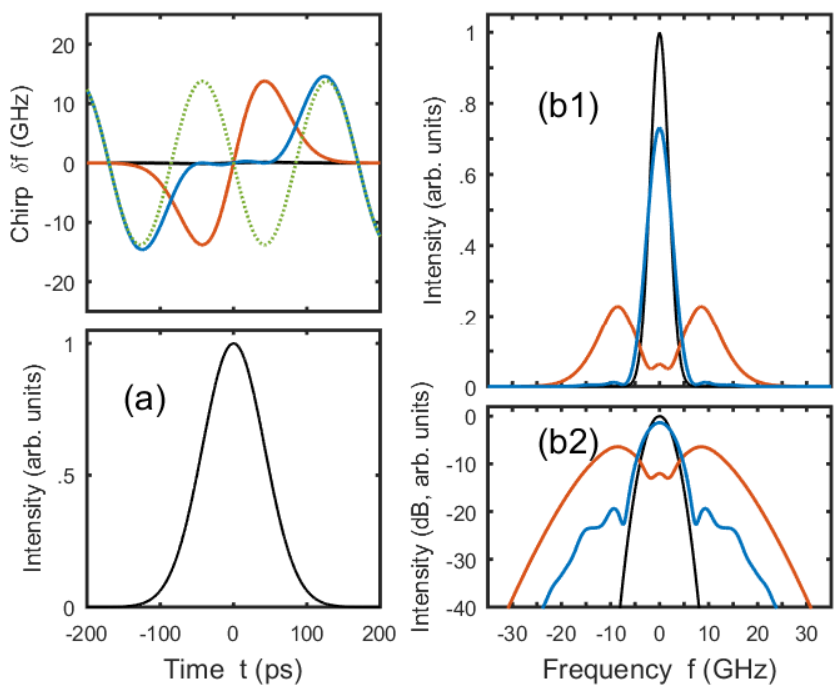

Figure 1: Impact of phase correction on a 100-ps Gaussian pulse after undergoing SPM in a fiber as obtained by numerical integration of Eq. (1): (a) temporal intensity and chirp profiles, and (b) spectral intensity profile on linear and logarithmic scales at the entrance (black) and the exit of the fiber before (red) and after (blue) phase compensation by a sinusoidal signal with frequency and amplitude chosen based on Eq. (2). The $B$-integral accumulated by the pulse is $B=2 \pi \mathrm{rad}$. The corrective chirp $\delta \omega_{\mathrm{c}}$ is plotted with a green dotted line. 
It is also interesting to represent the pulse electric field on the complex plane $(I(t) ; \phi(t))$ (Fig. 2). While the pulse affected by SPM traces a curve similar to an Archimedean spiral $(I(t) ; B I(t))$ (red curve), we can readily see from the graph that after phase compensation the phase is nearly constant over most of the pulse (blue solid curve), indicating a close-to-Fourier-transform-limited profile. On the contrary, higher values of the accumulated $B$-integral entail stronger deviations from the ideal profile (blue dashed curve).

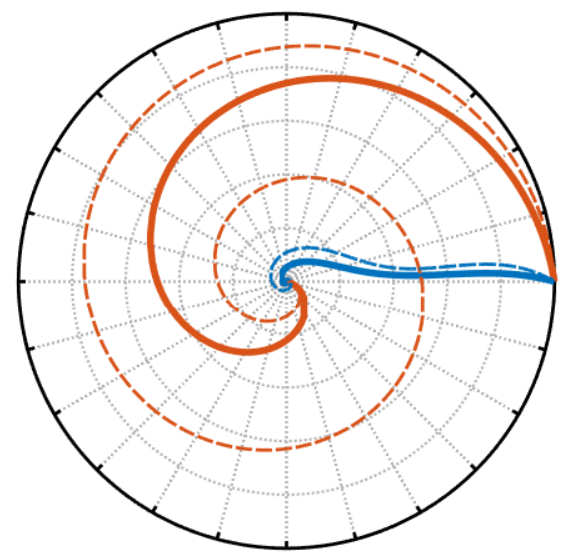

Figure 2: Polar graph $(I(t) ; \phi(t))$ of the electric field of a 100 ps Gaussian pulse after undergoing SPM in a fiber before (red) and after (blue) phase compensation by a sinusoidal signal with frequency and amplitude chosen based on Eq. (2). The results obtained for the $B$-integral values $B=2 \pi \mathrm{rad}$ and $B=4 \pi \mathrm{rad}$ are plotted with thick solid lines and thin dashed lines, respectively.

\section{SPM-compensation capability and analytical results}

We have numerically assessed the ability of our method to counteract SPM over a wide range of values of the $B$-integral accumulated in the fiber. The results obtained for the initially 100 -ps-wide Gaussian pulse and the corrective phase modulation specified in the previous section are summarized in Fig. 3, and highlight that the corrective approach can provide mitigation of SPM up to very high $B$ values: the fwhm of the pulse spectrum is largely preserved and no spectral ripple develops. However, as already seen in Fig. 2, the cancellation of the nonlinear phase is not complete and the intensity level of spectral side lobes tends to grow with increasing accumulated $B$-integral. This is also reflected in the evolution of the root-mean-square (rms) spectral width of 
the pulse with the $B$-integral (Fig. 3(b)): the rms spectrum broadening is reduced by a factor close to 4 relative to the case when no phase correction is used but it experiences a continuous increase with $B$ due to growing spectral side-lobes. The same trend is observed in the evolution of the Strehl ratio of the pulse: while this remains well above 0.5 , it slowly deteriorates with the increase of $B$. An exact formula for the rms spectral width of the pulse is derived hereafter.

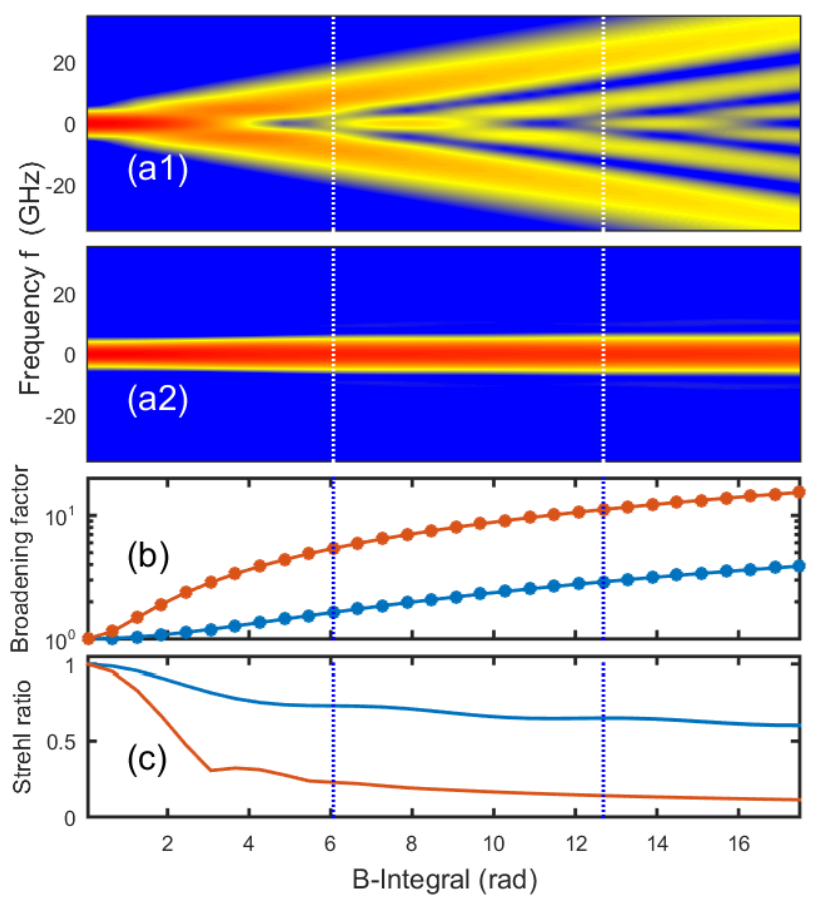

Figure 3: (a) Numerically computed evolution of the spectrum (logarithmic scale, $20 \mathrm{~dB}$ dynamics) of an initial $100 \mathrm{ps}$ Gaussian pulse after undergoing SPM in a fiber with the accumulated $B$-integral, before (subplot 1 ) and after (subplot 2) phase compensation by a sinusoidal signal with frequency and amplitude chosen based on Eq. (2). (b)-(c) Evolutions of the rms spectral broadening factor and the Strehl ratio of the pulse at the exit of the fiber. The results obtained without and with phase correction are plotted with red and blue lines, respectively. The vertical dotted lines indicate the $B$-integral values used in Figs. 1 and 2. The red and blue circles represent the analytical predictions from Eq. (5) and Eq. (9), respectively. 


\subsection{Spectral width calculation}

Following the calculation described by S. C. Pinault and M. J. Potasek in (Pinault,Potasek 1985) and used by us in (Boscolo et al. 2017a), we compute the rms spectral width $\sigma_{\omega}$ by computing the variance

$$
\sigma_{\omega}^{2}=\left\langle\omega^{2}\right\rangle-\langle\omega\rangle^{2}=\frac{\int \omega^{2}|\tilde{\psi}(\omega)|^{2} d \omega}{\int|\tilde{\psi}(\omega)|^{2} d \omega}-\left[\frac{\int \omega|\tilde{\psi}(\omega)|^{2} d \omega}{\int|\tilde{\psi}(\omega)|^{2} d \omega}\right]^{2}
$$

where $\tilde{\psi}(\omega)$ is the Fourier transform of the pulse envelope $\psi(t)$. Using Fourier-transform and convolution theorems in a combination with integration by parts, $\sigma_{\omega}^{2}$ can be expressed in terms of $\psi(t):$

$$
\sigma_{\omega}^{2}=\frac{\int\left|\psi^{\prime}(t)\right|^{2} d t}{\int|\psi(t)|^{2} d t}+\left[\frac{\int \psi^{\prime}(t) \psi^{*}(t) d t}{\int|\psi(t)|^{2} d t}\right]^{2}
$$

where' and * denote derivation and complex conjugation, respectively.

In the case of a Gaussian pulse affected by SPM, the closed-form evaluation of the integrals above yields (Pinault,Potasek 1985) :

$$
\frac{\sigma_{\omega}}{\sigma_{\omega 0}}=\sqrt{1+\frac{4 \cdot B^{2}}{3 \sqrt{3}}} \approx \sqrt{1+(0.8774 \cdot B)^{2}}
$$

where $\sigma_{\omega 0}$ is the initial rms spectral width of the pulse. Note that $\sigma_{\omega} \approx 0.8774 \cdot B \cdot \sigma_{\omega 0}$ for $B \square 1$. When a sinusoidal temporal phase modulation is imposed on the pulse, we obtain:

$$
\frac{\sigma_{\omega C}}{\sigma_{\omega 0}}=\left(\begin{array}{c}
1+\frac{4 \cdot B^{2}}{3 \sqrt{3}}+\sqrt{2} \cdot A_{C} \cdot B \cdot \omega_{C}^{2} \cdot T_{0}^{2} \cdot \exp \left(-\frac{\omega_{C}^{2} T_{0}^{2}}{8}\right) \cdot \cos \left(\phi_{0}\right) \\
\quad+A_{C}^{2} \cdot \omega_{C}^{2} \cdot T_{0}^{2} \cdot\left(1-e^{-\frac{\omega_{C}^{2} T_{0}^{2}}{2}}\right) \cdot\left(1+e^{-\frac{\omega_{C}^{2} T_{0}^{2}}{2}} \cos \left(2 \phi_{0}\right)\right)
\end{array}\right)^{\frac{1}{2}}
$$


This, with the choice $\phi_{0}=\pi$, reduces to:

$$
\frac{\sigma_{\omega C}}{\sigma_{\omega 0}}=\left(1+\frac{4 \cdot B^{2}}{3 \sqrt{3}}-\sqrt{2} A_{C} \cdot B \cdot \omega_{C}^{2} \cdot T_{0}^{2} \cdot \exp \left(-\frac{\omega_{C}^{2} T_{0}^{2}}{8}\right)+A_{C}^{2} \cdot \omega_{C}^{2} \cdot T_{0}^{2} \cdot\left(1-e^{-\omega_{C}^{2} T_{0}^{2}}\right)\right)^{\frac{1}{2}}
$$

It is notable that if we set $\omega_{C}=\omega_{C T G}=\pi /\left(\sqrt{2} T_{0}\right)$ in Eq. (7), we can find the optimum amplitude of the modulation $A^{\prime}{ }_{C T G}$ that minimizes the rms spectrum broadening generated by SPM by a derivative test:

$$
\left.\frac{\partial}{\partial A_{C}}\left(\frac{\sigma_{\omega C}^{2}}{\sigma_{\omega 0}^{2}}\right)\right|_{A_{C}=A_{C T G}^{\prime}}=0, \text { yielding } A_{C T G}^{\prime}=\frac{B}{\sqrt{2}} \frac{\mathrm{e}^{-\pi^{2} / 16}}{1-\mathrm{e}^{-\pi^{2} / 2}} \approx 0.384 \cdot B
$$

This amplitude value is in excellent agreement (less than 0.5 percent change) with the value $A_{C T G}$ given in Eq. (2), which was obtained through a more natural analysis. Equation (8) also confirms that as long as the propagation regime in the fiber is purely nonlinear, the duration of the initial pulse does not affect the amplitude of the corrective phase. Now, when we substitute Eq. (2) into Eq. (7), we obtain:

$$
\frac{\sigma_{\omega C}}{\sigma_{\omega 0}}=\left(1+\frac{4 \cdot B^{2}}{3 \sqrt{3}}-\frac{2 \cdot B^{2}}{e^{1 / 2}}\left(\frac{\pi}{\sqrt{2}} e^{-\frac{\pi^{2}}{16}}-\frac{2}{e^{1 / 2}}\left(1-e^{-\frac{\pi^{2}}{2}}\right)\right)\right)^{\frac{1}{2}} \approx \sqrt{1+(0.2146 \cdot B)^{2}}
$$

The rms spectral width evolutions with the accumulated $B$-integral predicted by Eqs. (5), (9) are in perfect agreement with the results of numerical simulations (Fig. 3(b)), thus confirming that for a 100 ps pulse the propagation is dominated by SPM. These predictions can be used to draw some interesting conclusions: while both evolutions follow the same law $\left(1+(k B)^{2}\right)^{1 / 2}$, the growth factor $k$ is approximately four times smaller when the phase correction is applied. This brings about a close to fourfold reduction in spectrum broadening at large $B: \sigma_{\omega} / \sigma_{\omega C} \approx 0.8774 / 0.2146$ for $B>1$, which explains the numerically observed trend in Fig. 3(b). 


\section{Impact of initial pulse shape}

In the previous sections, we have illustrated our proposed concept with a Gaussian input pulse and showed that an additional sinusoidal modulation of the temporal phase is remarkably effective at lessening the spectrum broadening induced by SPM. However, it is well known that SPM is highly sensitive to the initial pulse shape, and one may wonder whether our approach works equally well with other widely used pulse shapes. Our phase compensation method is in fact capable of effectively counteracting the unwanted effects of SPM on the propagation of other pulse waveforms, provided that these can be fitted by a parabolic profile in their central part (Boscolo et al. 2017a). To corroborate this statement, here we illustrate the case of a hyperbolic secant pulse like those delivered by soliton lasers. Following the same idea of reducing the chirp in the central region of the pulse and a similar calculation, one may derive the analogue of the formulae presented in Eq. (2) for the pulse intensity profile $I_{S}(t)=\operatorname{sech}^{2}\left(T / T_{0}\right)$ :

$$
\left\{\begin{array}{l}
\omega_{C T S}=\pi /\left(T_{0} \ln \left(\frac{\sqrt{3}+1}{\sqrt{3}-1}\right)\right) \approx 2.385 / T_{0} \\
A_{C T S}=\frac{4 \cdot B \cdot 3^{-3 / 2}}{\pi} \ln \left(\frac{\sqrt{3}+1}{\sqrt{3}-1}\right) \approx 0.322 \cdot B
\end{array}\right.
$$

Figure 4 illustrates the impact of a sinusoidal phase modulation with the frequency and amplitude chosen based on Eq. (10) on a 100 ps hyperbolic secant pulse after it undergoes SPM in the fiber as obtained by numerical integration of Eq. (1). We note that in the case of a purely nonlinear propagation, an analytic expression for the SPM-broadened spectrum of a hyperbolic secant pulse can be obtained (Phan Huy et al. 2010). We can see in Fig. 4 that the parameter choice of Eq. (10) achieves fairly good cancellation of the chirp of the pulse near the pulse center. The flat phase achieved around the peak intensity is also visible in the polar graph of Fig. 5. However, the deviations from perfect phase compensation on the pulse wings are rather strong, and these result in strong side lobes in the spectrum. Accordingly, despite the considerable enhancement contributed by the phase correction, the Strehl ratio of the pulse remains below 0.5. 

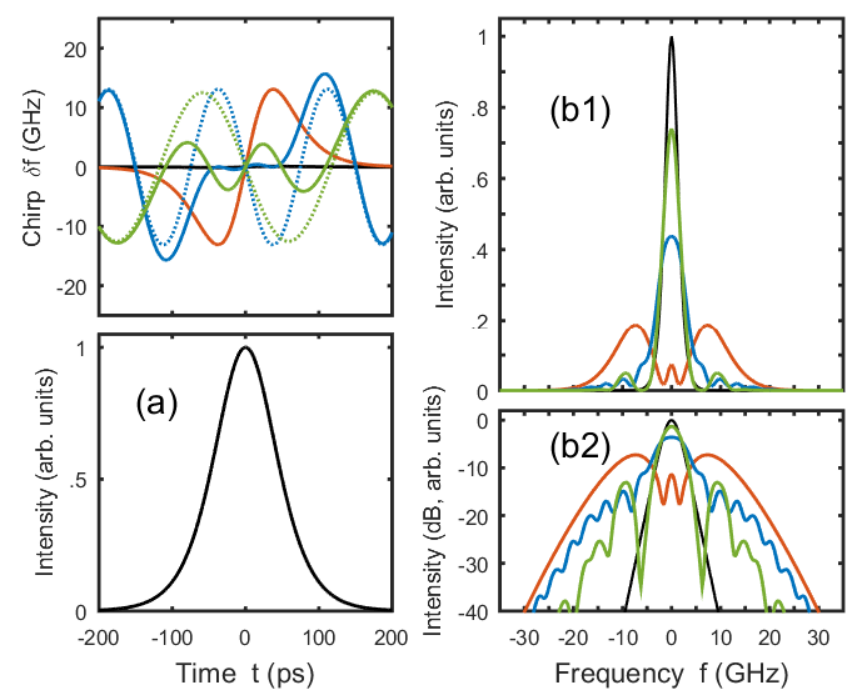

Figure 4: Impact of phase correction on a 100 ps hyperbolic secant pulse after undergoing SPM in a fiber as obtained by numerical integration of Eq. (1): (a) temporal intensity and chirp profiles, and (b) spectral intensity profile on linear and logarithmic scales at the entrance (black) and the exit of the fiber before (red) and after phase compensation by a sinusoidal signal with frequency and amplitude chosen based on Eq. (10) (blue) and with optimized frequency and amplitude (green). The $B$-integral accumulated by the pulse is $B=2 \pi \mathrm{rad}$. The corrective chirp $\delta \omega_{\mathrm{c}}$ resulting from Eq. (10) and from the optimized modulation parameters are plotted with blue and green dotted lines, respectively.

In order to improve our results, we have used an iterative procedure to find the best parameters of the modulating sinusoid: starting from the values $\omega_{C T S}$ and $A_{C T S}$ provided by Eq. (10), we have scanned the values close to this initial guess and selected the combination that yielded the highest Strehl ratio. The results obtained with the optimized parameters are plotted in Fig. 4 with green lines. With this strategy, the Strehl ratio has increased up to 0.74 and the frequency extent of the spectral side lobes has lessened. As we have observed in our previous work relating to nonlinear spectral compression (Boscolo et al. 2016), a constructive interference between different pulse parts having the same instantaneous frequency is favorable for attaining high Strehl ratios. This however occurs at the expense of a slight increase in the intensity level of the spectral side lobes. We would like to emphasize that, contrary to the parameter set of Eq. (10), the optimized modulation parameters do not achieve cancellation of the chirp near the pulse center. This is also readily seen in Fig. 5: the phase of the pulse is far from the Fourier transform limit. Hence, such choice of modulation parameters would make the phase correction method unsuitable for application in the context of phase regeneration of phase-encoded telecommunication signals. 


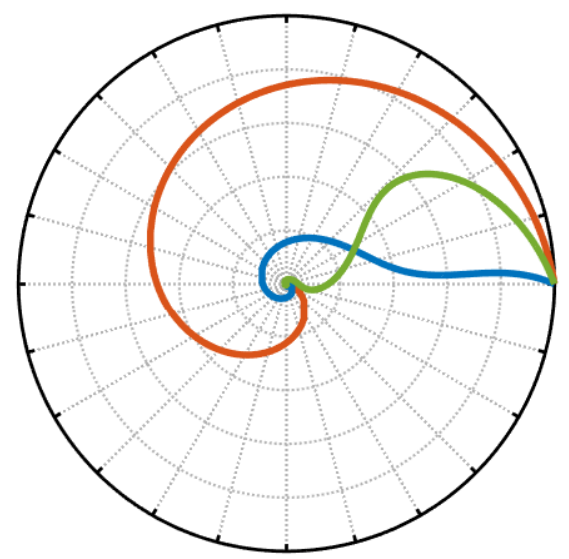

Figure 5: Polar graph $(I(t) ; \phi(t))$ of the electric field of a 100 ps hyperbolic secant pulse after undergoing SPM in a fiber before (red) and after phase compensation by a sinusoidal signal with frequency and amplitude chosen based on Eq. (10) (blue) and with optimized frequency and amplitude (green). The accumulated $B$-integral is $B=2 \pi$.

Figure 6 and 7 summarize the performance of the phase compensation method using optimized parameters across a wide range of $B$ values, and highlight an excellent mitigation of SPM. We can see in Fig. 6 that with the application of the corrective phase modulation, there is no noticeable spectrum broadening in terms of fwhm extension with increasing nonlinear phase shift accumulated in propagation while only a moderate broadening is observed in terms of rms width. However, the presence of side lobes in the spectrum is apparent. Figure 7 shows that the optimized sinusoidal modulation triggers a threefold decrease in the rms spectrum broadening generated from SPM compared to the modulation based on Eq. (10), and the Strehl ratio remains above 0.5 for $B$ values as high as $17 \mathrm{rad}$. The frequency and amplitude of the modulating sinusoid are plotted as functions of the $B$-integral in panels (c) and (d) of Fig. 7. We can note that the optimum frequency is significantly lower than the frequency $\omega_{\text {CTS }}$ defined in Eq. (10) and, contrary to $\omega_{C T S}$, it evolves with the accumulated nonlinear phase shift. It is noteworthy that with the range of modulation frequencies being studied, it is possible to drive the phase modulator with the master clock that has been used in (Ulmer et al. 2016) to generate RZ50-like signals. The optimum amplitude of the phase modulation is slightly higher than the value $A_{C T S}$ from Eq. (10), while it features a similar linear variation with the $B$-integral. From a practical viewpoint, it is important to notice that the choice of $\omega_{C}$ is not highly critical and an unoptimized value of $\omega_{C}$ can be partly compensated for by a change of $A_{C}$. 


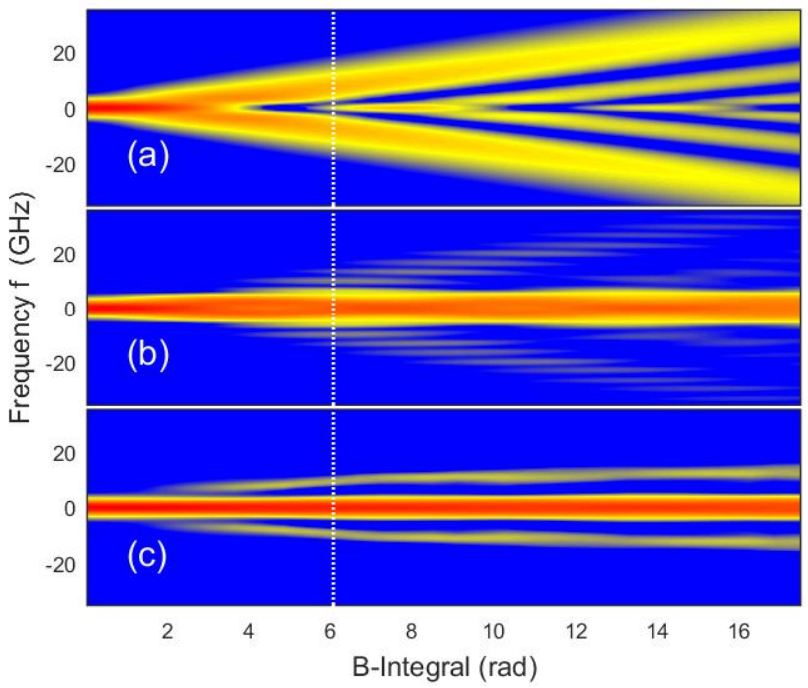

Figure 6: Numerically computed evolution of the spectrum (logarithmic scale, $20 \mathrm{~dB}$ dynamics) of an initial $100 \mathrm{ps}$ hyperbolic secant pulse after undergoing SPM in a fiber with the accumulated $B$-integral, before (subplot a) and after phase compensation by a sinusoidal signal with frequency and amplitude chosen based on Eq. (10) (subplot b) and with optimized frequency and amplitude (subplot c). The vertical dotted line indicates the $B$-integral value used in Figs. 4 and 5. 


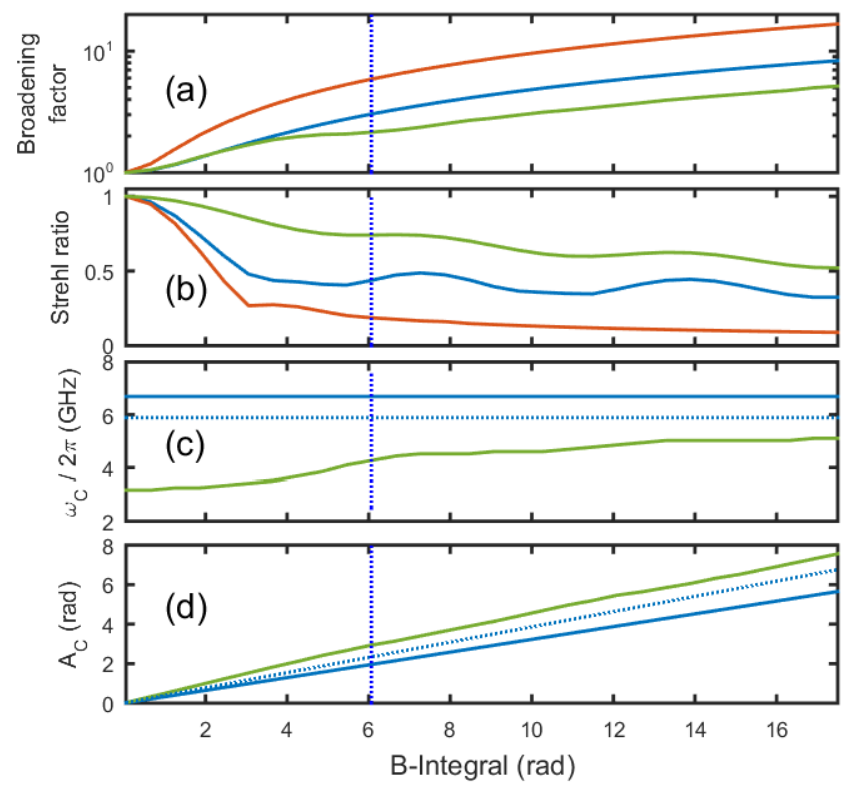

Figure 7: Numerically computed evolutions of (a) the rms spectral broadening factor and (b) the Strehl ratio of an initial 100 ps hyperbolic secant pulse after undergoing SPM in a fiber with the accumulated B-integral. (c)-(d) Evolutions of the frequency and amplitude of the sinusoidal phase modulation. The results obtained without phase modulation, with the phase modulation chosen based on Eq. (10), and with the optimized phase modulation are plotted with red, blue and green lines, respectively. The dotted blue lines in subplots (c) and (d) represent the modulation frequency and amplitude used for a Gaussian pulse based on Eq. (2). The vertical dotted lines indicate the $B$-integral value used in Figs. 4 and 5. 


\section{Impact of initial temporal pulse duration}

So far we have described the application of our SPM-mitigation method to optical pulses propagating through an optical fiber in the nonlinearity-dominant regime. However, it is well known that for long fiber lengths and/or short pulse durations, the effects of dispersion on propagation become important. The aim of this section is to assess the impact of the temporal duration of the initial pulses on the performance of our technique. To this end, we have varied the fwhm duration of a Gaussian input pulse from a few to a hundred picoseconds, and studied the compensation of the SPM acquired by the pulse upon propagation in a highly nonlinear fiber with the same parameters as those given in Sec. 2. Figure 8 gives a characterization of the pulse after accumulating a $B$-integral of $2 \pi$ rad in the fiber as a function of its initial temporal duration in both the frequency domain - through the evaluation of the rms spectral broadening factor and Strehl ratio - and the time domain - through the evaluation of the rms pulse width and excess kurtosis parameter (DeCarlo 1997). First, we observe that initial temporal durations above 30 ps do not affect either the spectral or the temporal metrics of the pulse, which pinpoints unaltered quality of the spectrum and characteristics of the temporal intensity profile for these pulse durations. In particular, the excess kurtosis is null and the duration of the output pulse equals the initial duration. This proves that the regime of propagation is dominated by Kerr nonlinearity with no influence of dispersion, and hence nonlinearity can be treated as being a lumped temporal phase modulator.

The picture is rather different for shorter initial pulse durations: the width of the pulse spectrum at the exit of the fiber increases significantly while the SPM-induced spectrum broadening diminishes slightly with decreasing initial duration. Besides, we can note a fall in effectiveness of the corrective phase modulation chosen based on Eq. (2) when it is applied as a post-compensation (blue solid line). For pulse durations shorter than $5 \mathrm{ps}$, the spectrum after phase compensation is even wider than the uncompensated case, highlighting the ineffectiveness of the design rules of Eq. (2) when the fiber dispersion comes into play. Similarly, the Strehl ratio of the pulse after phase compensation drops with decreasing initial duration. These features are ascribed to the changes experienced by the temporal intensity profile of the pulse: the combination of normal GVD and SPM makes the pulse temporally broaden and change shape toward a parabolic (Anderson et al. 1992; Finot et al. 2007) and then almost rectangular pulse form. This is evidenced by the excess kurtosis parameter approaching -1 . We note that accurate analytical formulae describing the evolution of the temporal and spectral rms widths and of the kurtosis of a pulse 
propagating in a nonlinear dispersive fiber are available (Burgoyne et al. 2007; Rosenberg et al. 2007) and may form the basis for the further development of design guidelines for our SPMcompensation scheme in the presence of dispersion. It is also worth mentioning that in the propagation regime being studied the nonlinearity still prevails over dispersion. Conversely, for longer propagation distances in the fiber, the broadening of the pulse spectrum may saturate and the pulse may enter spectronic dynamics (Zeytunyan et al. 2009; Castelló-Lurbe et al. 2013; Parriaux et al. 2017).

We have optimized the parameters of the sinusoidal phase modulation applied as postcompensation for each value of the initial pulse duration using the same iterative procedure as described in the previous section. The results are plotted in Fig. 8 with green lines, and show that an almost complete compensation of the SPM at any pulse duration is achievable by use of this approach. We will return to this point below. Furthermore, the effectiveness of the approach becomes more pronounced when shorter durations are involved.

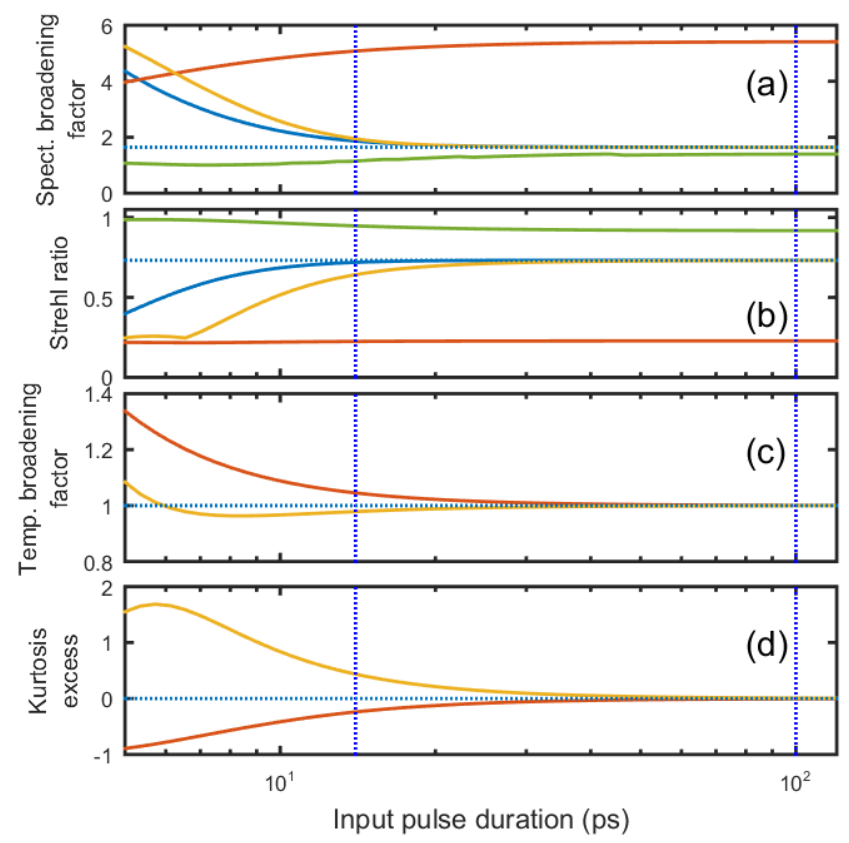

Figure 8: Numerically computed evolutions of (a) the rms spectral broadening factor, (b) the Strehl ratio, (c) the rms temporal broadening factor, and (d) the excess kurtosis parameter of an initial Gaussian pulse after undergoing SPM in a fiber with the input pulse duration. The accumulated $B$-integral is $B=2 \pi$ The results obtained without phase modulation, with the phase modulation chosen based on Eq. (2) and applied as a post-compensation, with the phase modulation chosen based on Eq. (2) and applied as a pre-compensation, and with the optimized phase modulation 
applied as a post-compensation are plotted with red, blue, yellow and green lines, respectively. The dotted blue lines recall the results obtained with a Gaussian pulse propagating in the quasi-pure nonlinear regime and a postcompensation based on Eq. (2). The vertical dotted lines indicate the temporal durations used in Figs. 1 and 7 and in Figs. 9 and 11.

For short pulse durations the interaction between nonlinearity and dispersion in the fiber is no longer negligible and so the pulse dynamics becomes more complex than the development of a simple temporal phase term. It is relevant here to compare the results that can be obtained by imparting the sinusoidal phase modulation before or after propagation in the fiber. Indeed, while for a purely nonlinear propagation the disposition of the nonlinear element and the external phase modulator does not impact the overall SPM-mitigation process, this is no longer the case when dispersion becomes important. In practice, while post-compensation has shown to be effective when it is done in the receiver of a telecommunication system (Xu et al. 2002), applications dealing with high-power pulses may favor a pre-compensation scheme to avoid damages to the optoelectronic component due to excessive optical power and mitigate the deleterious Brillouin backscattering (Ulmer et al. 2016; Su et al. 2013). It is noteworthy that with the phase modulation applied as a pre-compensation, the process can be reinterpreted as spectral focusing with the nonlinear phase accumulated by the pulse in propagation compensating an initial and opposite phase modulation (Zohrabian,Mouradian 1995; Oberthaler,Höpfel 1993): the spectrum is broadened by the pre-compensation, and the SPM process then compresses it back to its original shape. These pulse dynamics are in some ways analogous to the dynamics experienced by a pulse while it undergoes SPM-based spectral compression in a fiber (Oberthaler,Höpfel 1993; Markaryan et al. 1991; Planas et al. 1993; Finot,Boscolo 2016). However, in the case of a phase pre-compensation, the temporal intensity profile of the pulse does not evolve towards a parabolic shape; on the contrary, it experiences an increase of its kurtosis and becomes leptokurtic of a similar kind as a triangular profile (Boscolo et al. 2008). The results that we have obtained by applying the sinusoidal modulation chosen based on Eq. (2) as a pre-compensation (yellow lines in Fig. 8) are even worse than those obtained within the post-compensation scheme, thus emphasizing that the design rules of Eq. (2) are not appropriate for an efficient mitigation of the SPM of short pulses. 
Now we focus on initial pulses having a fwhm duration of $14 \mathrm{ps,} \mathrm{for} \mathrm{which} \mathrm{a} \mathrm{sinusoidal}$ modulation based on Eq. (2) can be generated by a 40-GHz phase modulator; such devices are widely commercially available. The pulse characterization showed in Fig. 9 obtained for an accumulated $B$-integral of $2 \pi$ rad substantiates the trends already captured by Fig. 8: for the pulse duration being considered, the temporal intensity profile of the pulse is affected by propagation in the fiber and the changes depend on whether the phase correction is applied before or after propagation. The quality of the SPM-mitigation highly depends on the compensation scheme being used. A pre-compensation with parameters chosen according to Eq. (2) yields poor mitigation. Optimizing the parameters of the sinusoidal modulation may help increase the spectral peak brightness and approach performances similar to those achieved with a post-compensation based on the parameters of Eq. (2). However, much better results both in terms of Strehl ratio enhancement and suppression of the residual side lobes in the spectrum can be obtained using a post-compensation with optimized parameters. The remarkable SPM-mitigation afforded by this approach is also readily apparent from the polar graph of Fig. 10, which highlights a notable reduction of the maximum phase of the pulse.
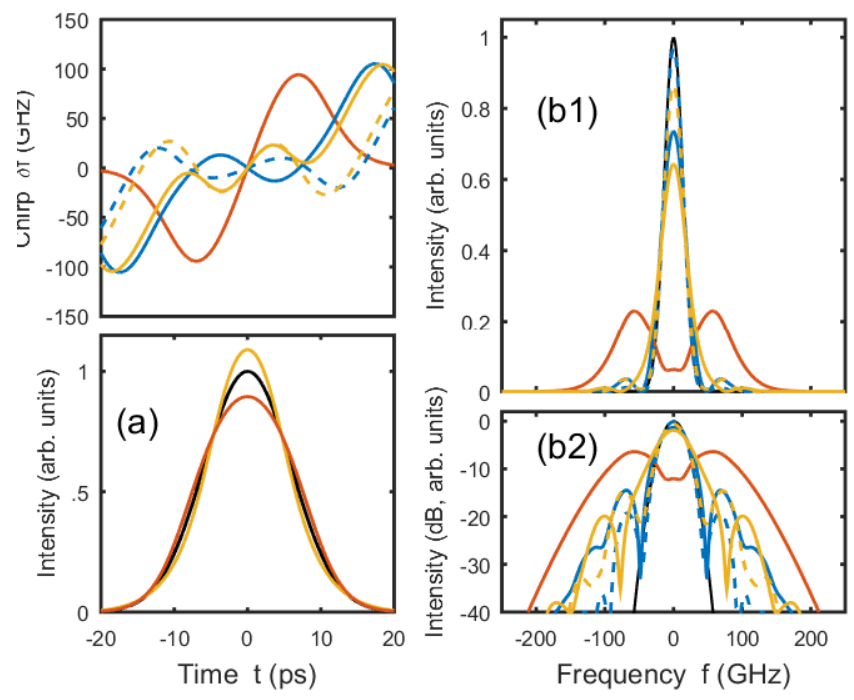

Figure 9: Impact of phase correction on a 14 ps Gaussian pulse after undergoing SPM in a fiber as obtained by numerical integration of Eq. (1): (a) temporal intensity and chirp profiles, and (b) spectral intensity profile on linear and logarithmic scales at the entrance (black) and the exit of the fiber without (red) and with phase compensation by a sinusoidal signal with frequency and amplitude chosen based on Eq. (2) (solid) and with optimized frequency and amplitude (dashed). The results obtained with a pre-compensation scheme (yellow) are compared with those obtained with a post-compensation scheme (blue). The $B$-integral accumulated by the pulse is $B=2 \pi \mathrm{rad}$. 


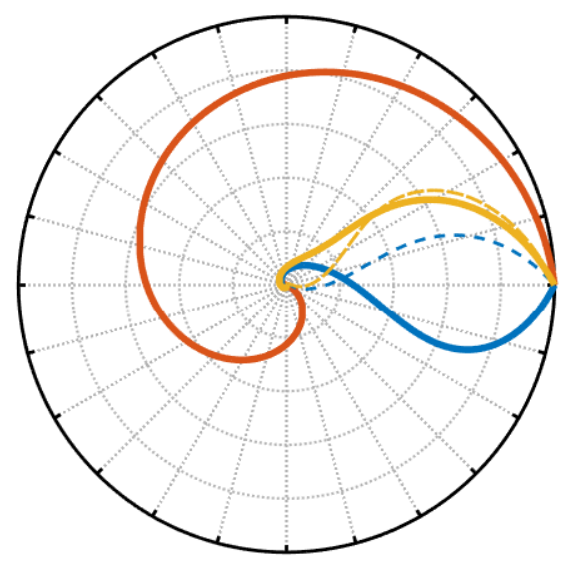

Figure 10: Polar graph $(I(t) ; \phi(t))$ of the electric field of a 14 ps Gaussian pulse after undergoing SPM in a fiber without phase compensation (red), with phase pre-compensation (yellow) and with phase post-compensation (blue). The results obtained with the sinusoidal modulation chosen based on Eq. (2) (solid) are compared with those obtained with the optimized sinusoidal modulation (dashed). The accumulated $B$-integral is $B=2 \pi$.

Further, we have tested the compliance of these observations with higher levels of SPM experienced by the $14 \mathrm{ps}$ input pulse upon propagation in the fiber. The results are summarized in Fig. 11 and indeed confirm the trends discussed above: the evolutions of the Strehl ratio and the spectral broadening factor evidence that the use of Eq. (2) within a pre-compensation scheme is inefficient. The use of optimized parameters for the sinusoidal modulation applied as a precompensation achieves performances similar to those of a post-compensation based on Eq. (2). Applying the sinusoidal modulation with optimized parameters as a post-compensation provides a nearly perfect SPM cancellation with a Strehl ratio that approaches 1 and an rms spectral width after fiber propagation that nearly equals the initial width. It is remarkable that the effectiveness of this approach does not seem to be impaired by the level of SPM accumulated by the pulse and the compensation process appears to work perfectly for $B$-integral values as high as 16 rad. This quite counterintuitive behavior can in fact be explained by the linearization of the temporal phase accumulated by the pulse upon propagation that is contributed by normal GVD (Anderson et al. 1992; Rosenberg et al. 2007). We can also see in Fig. 11(c) that the frequency of the sinusoidal modulation does not change significantly with the level of accumulated SPM. The optimized frequency is threefold smaller than the frequency chosen based on Eq. (2), thereby being more easily attainable with current optoelectronic components. The optimized amplitude of the 
modulation is 20 percent larger than the amplitude given by Eq. (2) (Fig. 11(d)) but yet remains fully accessible with current technology (Godil et al. 1994; Plansinis et al. 2015).

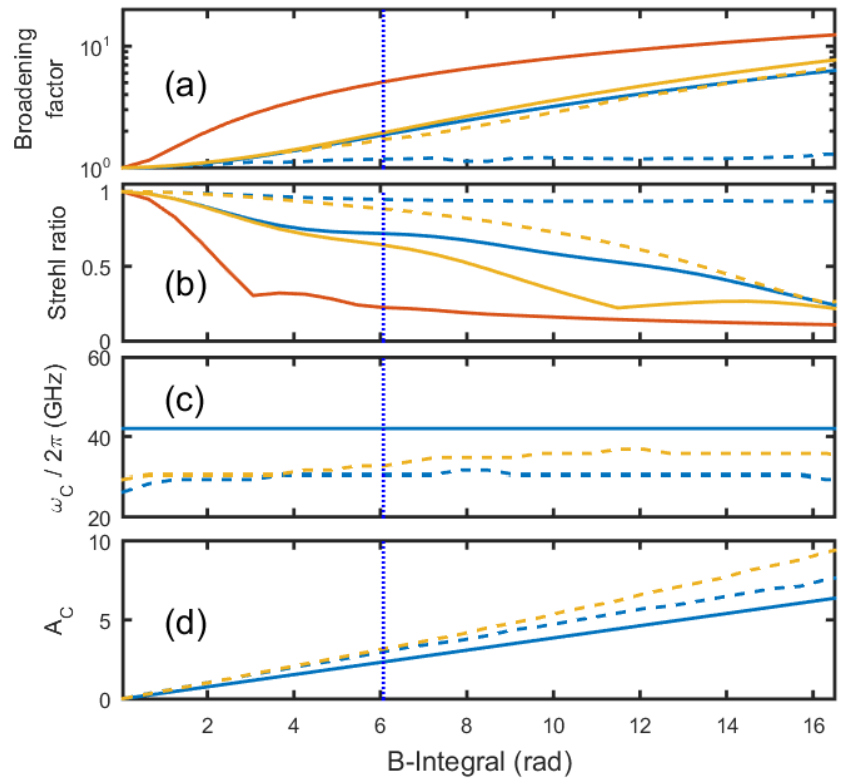

Figure 11: Numerically computed evolutions of (a) the rms spectral broadening factor and (b) the Strehl ratio of an initial 14 ps Gaussian pulse after undergoing SPM in a fiber with the accumulated B-integral. (c)-(d) Evolutions of the frequency and amplitude of the sinusoidal phase modulation. The results obtained without phase modulation, with the phase modulation applied as a pre-compensation and with the phase modulation applied as a post-compensation are plotted with red, yellow and blue lines, respectively. The results obtained with the sinusoidal modulation chosen based on Eq. (2) (solid) are compared with those obtained with the optimized sinusoidal modulation (dashed). The vertical dotted lines indicate the $B$-integral value used in Figs. 9 and 10. 


\section{Conclusion}

We have presented an in-depth characterization of the mitigation of the impact of SPM on optical pulses propagating through an optical fiber relying on imparting a sinusoidal phase modulation to the pulses in the time domain. We have presented simple analytic guidelines for the choice of the frequency and amplitude of the modulation, and described a calculation that gives an exact closed formula for the rms spectral width of an initially Gaussian pulse after undergoing SPM and with the corrective phase applied, which confirms the remarkable reduction of the SPM-induced spectrum broadening attainable with the compensation approach. We have then extended our analysis to another commonly used pulse shape, hyperbolic secant pulses, and shown that for such pulses optimization of the parameters of the modulating sinusoid through a scan of the amplitudefrequency space outperforms the parameter choice based on the analytic guidelines. Further, we have assessed the impact of the temporal duration of the initial pulses on the effectiveness of the compensation technique, and emphasized the important role played by the fiber dispersion in the compensation process for short pulses: the interaction between SPM and GVD engenders significantly different performances depending on whether the sinusoidal modulation is applied as a pre- or post-compensation. Specifically, we have shown that remarkable SPM mitigation is attainable by use of a post-compensation based on optimized parameters. Handling pulses as short as 10 ps with our SPM-mitigation scheme is fully realistic by means of existing optoelectronic components, while the use of cross-phase modulation in the fiber could also be a solution (Mouradian et al. 2000). In this paper, we have focused on the regime of normal dispersion that is typical of fiber amplifiers, yet the use of an anomalously dispersive fiber may significantly change the conclusions drawn (Paré et al. 1999).

We believe that the present study can provide a deeper insight into the association of the SPM effects with an external sinusoidal phase modulation and can be of interest for multistage fiber amplifier architectures. We also expect that some features of the spectral compression process driven by SPM discussed in our previous work (Boscolo et al. 2017b) can be readily extended to the SPM-mitigation scheme described in this paper so that the present scheme should be rather stable against amplitude fluctuations and signal-to-noise ratio degradation of the input pulses. We also believe that, similarly to a nonlinear spectral compression stage, the present technique could be combined with fiber laser architectures into new cavity designs (Ilday,Wise 2002; Boscolo et 
al. 2012). Other prospected applications of the proposed approach include the reduction of different unwanted temporal phase defects such as those developed by pulses upon propagation in gain switched fibers or semiconductors devices. 


\section{Acknowledgements}

We thank Bertrand Kibler and Julien Fatome for very stimulating discussions about the initial concept. We also thank Todd Ulmer for bringing to our knowledge several important previous publications on the method being studied. We acknowledge financial support by the Région Bourgogne (Pari Photcom) and the Agence Nationale de la Recherche (Labex Action ANR-11LABX-01-01). This work was also supported by the European Union within the framework of the operational Program FEDER-FSE Bourgogne 2014-2020 and by the Institut Universitaire de France. 


\section{References}

Agrawal, G.P.: Nonlinear Fiber Optics, Fourth Edition. Academic Press, San Francisco, CA (2006)

Anderson, D., Desaix, M., Lisak, M., Quiroga-Teixeiro, M.L.: Wave-breaking in nonlinear optical fibers. J. Opt. Soc. Am. B 9(8), 1358-1361 (1992)

Audo, F., Boscolo, S., Fatome, J., Kibler, B., Finot, C.: Nonlinear spectrum broadening cancellation by sinusoidal phase modulation. Opt. Lett. 42(15), 2902-2905 (2017)

Audo, F., Finot, C.: Extinction ratio enhancement by sinusoidal phase modulation and nonlinear spectral focusing. Laser Physics 27, 115401 (2017)

Blanco-Redondo, A., Martijn De Sterke, C., Sipe, J.E., Krauss, T.F., Eggleton, B.J., Husko: Pure-quartic solitons. Nature Communications 7 (2016)

Boscolo, S., Chaussard, F., Andresen, E.R., Rigneault, H., Finot, C.: Impact of initial pulse shape on the nonlinear spectral compression in optical fibre. Opt. Laser Technol. 99, 301-309 (2017a)

Boscolo, S., Fatome, J., Finot, C.: Impact of amplitude jitter and signal-to-noise ratio on the nonlinear spectral compression in optical fibres. Opt. Commun. 389, 197-202 (2017b)

Boscolo, S., Latkin, A.I., Turitsyn, S.K.: Passive nonlinear pulse shaping in normally dispersive fiber systems. IEEE J. Quantum Electron. 44(12), 1196-1203 (2008)

Boscolo, S., Mouradian, L.K., Finot, C.: Enhanced nonlinear spectral compression in fibre by external sinusoidal phase modulation. J. Opt. 18(10), 105504 (2016)

Boscolo, S., Turitsyn, S.K., Finot, C.: Amplifier similariton fiber laser with nonlinear spectral compression. Opt. Lett. 37(21), 4531-4533 (2012). doi:10.1364/ol.37.004531

Burgoyne, B., Godbout, N., Lacroix, S.: Nonlinear pulse propagation in optical fibers using second order moments. Opt. Express 15(16), 10075-10090 (2007)

Castelló-Lurbe, D., Andrés, P., Silvestre, E.: Dispersion-to-spectrum mapping in nonlinear fibers based on optical wave-breaking. Opt. Express 21(23), 28550-28558 (2013). doi:10.1364/oe.21.028550

DeCarlo, L.T.: On the meaning and use of kurtosis. Psychol. Methods 2(3), 292-307 (1997)

Finot, C., Boscolo, S.: Design rules for nonlinear spectral compression in optical fibers. J. Opt. Soc. Am. B 33(4), 760-767 (2016)

Finot, C., Dudley, J.M., Kibler, B., Richardson, D.J., Millot, G.: Optical parabolic pulse generation and applications. IEEE J. Quantum Electron. 45(11), 1482-1489 (2009)

Finot, C., Provost, L., Petropoulos, P., Richardson, D.J.: Parabolic pulse generation through passive nonlinear pulse reshaping in a normally dispersive two segment fiber device. Opt. Express 15(3), 852-864 (2007)

Godil, A.A., Auld, B.A., Bloom, D.M.: Picosecond time-lenses. IEEE J. Quantum Electron. 30(3), 827837 (1994). doi:10.1109/3.286176

Hasegawa, A., Tappert, F.: Transmission of stationary nonlinear optical pulses in dispersive dielectric fibers. II. Normal dispersion. Appl. Phys. Lett. 23(4), 171-172 (1973)

Ilday, F.Ö., Wise, F.W.: Nonlinearity management: a route to high-energy soliton fiber lasers. J. Opt. Soc. Am. B 19(3), 470-476 (2002)

Ma, X., Zhu, C., Hu, I.N., Kaplan, A., Galvanauskas, A.: Single-mode chirally-coupled-core fibers with larger than 50 $\mu \mathrm{m}$ diameter cores. Opt. Express 22(8), 9206-9219 (2014). doi:10.1364/oe.22.009206

Markaryan, N.L., Muradyan, L.K., Papazyan, T.A.: Spectral compression of ultrashort laser pulses. Kvantovaya Elektron. 18(7), 865-867 (1991) 
Michieletto, M., Lyngsø, J.K., Jakobsen, C., Lægsgaard, J., Bang, O., Alkeskjold, T.T.: Hollow-core fibers for high power pulse delivery. Opt. Express 24(7), 7103-7119 (2016). doi:10.1364/oe.24.007103

Mouradian, L.K., Louradour, F., Messager, V., Barthélémy, A., Froehly, C.: Spectro-temporal imaging of femtosecond events. IEEE J. Quantum Electron. 36(7), 795-801 (2000)

Munroe, M.J., Hamamoto, M.Y., Dutton, D.A.: Reduction of SPM induced spectral broadening in a high peak power narrow linewidth IR fiber laser using phase modulation. In: 2009, pp. $71952 \mathrm{~N}-$ $71952 \mathrm{~N}-71956$

Oberthaler, M., Höpfel, R.A.: Spectral narrowing of ultrashort laser pulses by self-phase modulation in optical fibers. Appl. Phys. Lett. 63(8), 1017-1019 (1993)

Ouzounov, D.G., Ahmad, F.R., Müller, D., Venkataraman, N., Gallagher, M.T., Thomas, M.G., Silcox, J., Koch, K.W., Gaeta, A.L.: Generation of Megawatt optical solitons in hollow-core photonic band-gap fibers. Science 301, 1702-1704 (2003)

Paré, C., Villeneuve, A., LaRochelle, S.: Split compensation of dispersion and self-phase modulation in optical communication systems. Opt. Commun. 160(1), 130-138 (1999)

Parriaux, A., Conforti, M., Bendahmane, A., Fatome, J., Finot, C., Trillo, S., Picqué, N., Millot, G.: Spectral broadening of picosecond pulses forming dispersive shock waves in optical fibers. Opt. Lett. 42(15), 3044-3047 (2017). doi:10.1364/ol.42.003044

Phan Huy, M.C., Baron, A., Lebrun, S., Frey, R., Delaye, P.: Characterization of self-phase modulation in liquid filled hollow core photonic bandgap fibers. J. Opt. Soc. Am. B 27(9), 1886-1893 (2010). doi:10.1364/josab.27.001886

Pierrot, S., Salin, F.: Amplification and compression of temporally shaped picosecond pulses in $\mathrm{Yb}$ doped rod-type fibers. Opt. Express 21(17), 20484-20496 (2013)

Pinault, S.C., Potasek, M.J.: Frequency broadening by self-phase modulation in optical fibers. J. Opt. Soc. Amer. B 2(8), 1318-1319 (1985)

Planas, S.A., Pires Mansur, N.L., Brito Cruz, C.H., Fragnito, H.L.: Spectral narrowing in the propagation of chirped pulses in single-mode fibers. Opt. Lett. 18(9), 699-701 (1993)

Plansinis, B.W., Donaldson, W.R., Agrawal, G.P.: Spectral changes induced by a phase modulator acting as a time lens. J. Opt. Soc. Am. B 32(8), 1550-1554 (2015). doi:10.1364/josab.32.001550

Richardson, D.J., Nilsson, J., Clarkson, W.A.: High power fiber lasers: current status and future perspectives. J. Opt. Soc. Am. B 27(11), B63-B92 (2010)

Rosenberg, C.-K., Anderson, D., Desaix, M., Johannisson, P., Lisak, M.: Evolution of optical pulses towards wave breaking in highly nonlinear fibres. Opt. Commun. 273, 272-277 (2007)

Seghilani, M., Maram, R., Azaña, J.: Mitigating nonlinear propagation impairments of ultrashort pulses by fractional temporal self-imaging. Opt. Lett 42(4), 879-882 (2017). doi:10.1364/ol.42.000879

Shah, L., Liu, Z., Hartl, I., Imeshev, G., Cho, G.C., Fermann, M.E.: High energy femtosecond Yb cubicon fiber amplifier. Opt. Express 13(12), 4717-4722 (2005)

Stolen, R.H., Lin, C.: Self-phase modulation in silica optical fibers. Phys. Rev. A 17(4), 1448-1453 (1978)

Strickland, D., Mourou, G.: Compression of amplified chirped optical pulses. Opt. Commun. 56, 219221 (1985)

Su, R., Zhou, P., Ma, P., Lü, H., Xu, X.: High-peak-power, single-frequency, single-mode, linearly polarized, nanosecond all-fiber laser based on self-phase modulation compensation. Appl. Opt. 52(30), 7331-7335 (2013). doi:10.1364/ao.52.007331

Ulmer, T., Roth, J.M., Minch, J.R., Chang, J., Moores, J.D., Nowak, G., Walther, F.G.: Mitigation of Self-Phase Modulation in High-Peak-Power Lasercom Systems. In: Lasers Congress 2016 
(ASSL, LSC, LAC), Boston, Massachusetts, 2016/10/30 2016. OSA Technical Digest (online), p. LW3B.6. Optical Society of America

van Howe, J., Zhu, G., Xu, C.: Compensation of self-phase modulation in fiber-based chirped-pulse amplification systems. Opt. Lett 31(11), 1756-1758 (2006). doi:10.1364/ol.31.001756

Wang, Q.Z., Liu, Q.D., Liu, D., Ho, P.P., Alfano, R.R.: High-resolution spectra of self-phase modulation in optical fibers. J. Opt. Soc. Am. B 11(6), 1084-1089 (1994). doi:10.1364/josab.11.001084

Xu, C., Mollenauer, L., Liu, X.: Compensation of nonlinear self-phase modulation with phase modulators. Electron. Lett. 38(24), 1578-1579 (2002). doi:10.1049/el:20021050

Zeytunyan, A., Yesayan, G., Mouradian, L., Kockaert, P., Emplit, P., Louradour, F., Barthélémy, A.: Nonlinear-dispersive similariton of passive fiber. J. Europ. Opt. Soc. Rap. Public. 4, 09009 (2009)

Zhou, S., Kuznetsova, L., Chong, A., Wise, F.W.: Compensation of nonlinear phase shifts with thirdorder dispersion in short-pulse fiber amplifiers. Opt. Express 13(13), 4869-4877 (2005)

Zhu, G., Edinberg, J., Xu, C.: Nonlinear distortion free fiber-based chirped pulse amplification with selfphase modulation up to $2 \pi$. Opt. Express 15(5), 2530-2534 (2007). doi:10.1364/oe.15.002530

Zohrabian, A.V., Mouradian, L.K.: Compression of the spectrum of picosecond ultrashort pulses. Quantum Electron. 25(11), 1076 (1995) 